\title{
MicroRNA-21 inhibits SMAD7 expression through a target sequence in the 3 ' untranslated region and inhibits proliferation of renal tubular epithelial cells
}

\author{
LI LIN $^{1}$, HUA GAN ${ }^{1}$, HAN ZHANG $^{1}$, WEIXUE TANG ${ }^{2}$, YUE SUN ${ }^{1}$, XUEQIN TANG $^{1}$, \\ DEQUAN KONG ${ }^{3}$, JIAJUN ZHOU ${ }^{4}$, YUXUAN WANG ${ }^{1}$ and YANLIN ZHU ${ }^{1}$ \\ Departments of ${ }^{1}$ Nephrology, ${ }^{2}$ Experiment Center and ${ }^{3}$ Surgery, The First Affiliated Hospital of \\ Chongqing Medical University, Chongqing 400016; ${ }^{4}$ Department of Nephrology, The First \\ Affiliated Hospital of Wannan Medical College, Wuhu, Anhui 241100, P.R. China
}

Received November 18, 2013; Accepted May 16, 2014

DOI: $10.3892 / \mathrm{mmr} .2014 .2312$

\begin{abstract}
MicroRNAs (miRNAs) are a class of small non-coding single-stranded RNAs that regulate gene expression at the posttranscriptional level. Since the identification of miRNA, accumulating research has shown their involvement in numerous biological processes, including timing of developmental patterning, embryogenesis, cell differentiation, organogenesis, growth control and pathogenesis of human diseases. It is estimated that $>30 \%$ human genes may be regulated by miRNA, and that each miRNA can regulate $>100$ target mRNAs. The widespread and distinct expression pattern of miRNAs in normal and disease states has been extensively investigated in the context of human diseases. Due to the diversity of targets, it is challenging to identify the specific target genes and elucidate the biological function of a certain miRNAs. In the present study, it was confirmed that SMAD7 is a direct target of miR-21, and overexpression of miR-21 may inhibit the proliferation of rat renal tubular epithelial cells. These findings confirm the results of previous studies, which have demonstrated that miR-21 regulates the expression of SMAD7 protein. However, further investigation is required to determine whether miR-21 is involved in renal development and disease, particularly diabetic nephropathy.
\end{abstract}

\section{Introduction}

The identification of microRNA (miRNA) in the 1990s was prominent in biological research (1). miRNAs are a class of small non-coding single-stranded RNA, 21-25 nucleotides in

Correspondence to: Ms Hua Gan and Ms Han Zhang, Department of Nephrology, The First Affiliated Hospital of Chongqing Medical University, 1 Youyi Road, Chongqing 400016, P.R. China

E-mail: ghzxgckd@163.com

E-mail:imu333@sina.com

Key words: microRNA, microRNA-21, SMAD7, renal tubular epithelial cell length, with diverse biological functions $(2,3)$. miRNA has been implicated in regulating cell division, differentiation, development, oncogenesis, apoptosis and other processes through translational arrest and/or messenger RNA (mRNA) degradation (4-6). It has been shown that a single miRNA may be directly responsible for the repression of hundreds of proteins, and indirectly for the regulation of thousands of others (4). Abnormal expression of miRNA has been implicated in various diseases, including cancer, diabetes, and cardiovascular, neurodegenerative and psychological disorders (5-8). Despite the involvement of miRNA in numerous biological and pathological processes, the large number of candidate targets and cellular pathways, which are regulated by miRNAs, remain to be understood.

miRNA-21 (miR-21) is a small RNA, which is ubiquitously expressed in normal tissues and cells (9). Previous studies have predominantly focused on the association between miR-21 and tumors, since miR-21 has been consistently identified to be overexpressed in numerous tumor samples (10), including glioblastomas (11), lung $(12,13)$, stomach (14), prostate (14) and colon cancer $(15,16)$, and cholangiocarcinoma (17) samples.

miR-21 has numerous predicted target genes; however, only a few have been validated in an experimental study (18). Despite the widespread expression of miR-21 in normal tissues, little is known regarding its physiological functions and regulatory mechanisms (19).

Transforming growth factor (TGF) $\beta$ is a multifunctional growth factor that functions in the initiation and termination of tissue repair. TGF- $\beta$ is considered to be a key mediator in disease pathogenesis (20-22). SMAD7 is a negative regulator of Smad signaling and inhibits TGF- $\beta$ activity through the prevention of the phosphorylation of $\operatorname{Smad} 2 / 3(23,24)$. However, the association between TGF- $\beta$ and miR-21 in renal disease, in particular diabetic nephtropathy, remains to be elucidated.

The aim of the present study was to investigate the functional interaction between miR-21 and the TGF- $\beta$ signaling pathway, in order to eventually determine its involvement in diabetic nephropathy. 


\section{Materials and methods}

Cell lines and culture conditions. Rat renal tubular epithelial cells and HEK 293T cells were obtained from the Shanghai Academy Sciences Cell Bank (Shanghai, China). Cell lines were cultured in Dulbecco's modified Eagle's medium (DMEM) high glucose $(25 \mathrm{mmol} / \mathrm{l})$ and low glucose ( $5 \mathrm{mmol} / \mathrm{l}$ ), respectively (Gibco-BRL, Carlsbad, CA, USA). The low glucose group served as a control, while the high glucose group represented the environment of renal cells in a patient with diabetes. The media were supplemented with $10 \%$ fetal bovine serum (FBS; Gibco-BRL). All cells were grown in a humidified incubator in $5 \% \mathrm{CO}_{2}$ at $37^{\circ} \mathrm{C}$.

Bioinformatic analyses. TargetScan 4.1 (http://www. targetscan.org), PicTar (http://pictar.mdc-berlin.de/) and miRNA.org (http://www.microrna.org/), GenBank (http:// www.ncbi.nlm.nih.gov/gene/) online databases were used to predict the targets of miR-21. These three programs used a different algorithm to identify highly complementary sites of miRNA. SMAD7 was consistently identified as a target of miR-21 with all three programs (Fig. 1).

Dual luciferase reporter assay. Luciferase reporter gene constructs containing either the wild-type or mutated full-length 3' untranslated region (UTR) of SMAD7, were constructed using the luciferase reporter vector psi-CHECK2 (Promega Corporation, Madison, WI, USA). The following primers were used for site direct mutagenesis plasmid construction: Forward: 5'-CACACTTTAATGCGGTTCATTTTTCTAACTACAAAGGTTT-3'; and reverse: 5'-AGTTAGAAAAATGAACCGCATTAAAGTGTGAGCAT GCTCA-3' for MutSmad7. ATAAGCT was mutated to GCGGTTC. The cells were transiently cotransfected into 24-well plates using Lipofectamine ${ }^{\mathrm{TM}}$ reagent (Invitrogen Life Technologies, Carlsbad, CA, USA). Mutations to the seed region of miR-21 mimics, which can result in high levels of miR-21 expression, and miR-21 negative controls (NC) which have no function were compounded. These were cotransfected into HEKT cells with the WT or mutant 3'UTR SMAD7. Reporter assays were performed at $48 \mathrm{~h}$ posttransfection using the Dual-luciferase Reporter Assay system (E1910; Promega Corporation) according to manufacturer's instructions. All experiments were performed in triplicate and the standard deviation was calculated.

miR-21 transfection in renal tubular epithelial cells. Rat renal tubular epithelial cells were transfected with $1.2 \mu \mathrm{l}$ lentivirus overexpressing miR-21 or empty virus (Shanghai Jikai Gene Chemical Co., Ltd, Shanghai, China). Flow cytometry (FACSVantage; BD Biosciences, San Jose, CA, USA) was used $36 \mathrm{~h}$ after transfection in order to assess transfection efficiencies. Total RNA was then extracted from the cells and the miR-21 levels were assessed using reverse transcription-quantitative polymerase chain reaction (RT-qPCR).

Cell growth inhibition assay. Following transfection, the cells were seeded into 96 -well plates at a density of 10,000 cells/well. Four plates were used in total, and were incubated for $0,24,48$,
Smad7 5 ' ... UGCUCACACUUUAAUAUAAGCUA...

\section{miR-21 3' AGUUGUAGUCAGACUAUUCGAU}

Figure 1. Predicted miR-21 target site (bold) in the 3'UTR of SMAD7, using TargetScan 4.1 .

or $72 \mathrm{~h}$. The cellular proliferation was analyzed at each time point using an MTT Cell Proliferation Assay kit (Invitrogen Life Technologies) over four days.

$R T-q P C R$. Cells were collected following transfection for $72 \mathrm{~h}$ and total RNA was extracted using TRIzol ${ }^{\mathrm{TM}}$ reagent (Invitrogen Life Technologies). cDNA was synthesized from the extracted RNA using a First-strand cDNA Synthesis kit (Promega Corporation) according to the manufacturer's instructions. The miRNA reverse transcription-PCR primers for miR-21, and endogenous control U6RT were purchased from Applied Biosystems (Applied Biosystems, Invitrogen Life Technologies). The primer sequences used in the experiment were designed as follows: Forward: 5'-ACACTC CAGCTGGGTAGCTTATCAGACTGAT-3'; and reverse: 5'-ACTGGTGTCGTGGAGTCG-3' for Rno-mir-21. qPCR analysis was performed using an ABI PRISM ${ }^{\circledR} 7500$ Sequence Detection system (Applied Biosystems). The conditions for the PCR were as follows: $95^{\circ} \mathrm{C}$ for $5 \mathrm{~min}, 95^{\circ} \mathrm{C}$ for $15 \mathrm{sec}$ and $62^{\circ} \mathrm{C}$ for $30 \mathrm{sec}$, for a total of 40 cycles. Each sample was run in triplicate. The comparative threshold cycle (CT) method was used to evaluate the relative gene expression.

Western blot analysis. Western blot analysis was performed according to standard protocols. Briefly, the cells were solubilized in lysis buffer (containing posphatase inhibitors, protease inhibitors and PMSF) and the protein was extracted using a Protein Extraction reagent (Sigma, St. Louis, MO, USA). Protein concentrations were determined using a Bio-Rad Protein Assay kit (Bio-Rad, Hercules, CA, USA). The proteins were separated by SDS-PAGE and transferred to nitrocellulose membranes. Then the membranes were blocked and incubated overnight at $4^{\circ} \mathrm{C}$ with a rabbit anti-SMAD7 antibody (Abcam, Cambridge, MA, USA). The membranes were then washed and incubated with horseradish peroxidase-conjugated goat anti-rabbit secondary antibody (Abcam) at $37^{\circ} \mathrm{C}$ for two hours. Proteins were visualized by an enhanced chemiluminescence detection system (Amersham Pharmacia Biotech, Piscataway, NJ, USA). When required, the membranes were stripped and reprobed with $\beta$-actin, as an internal loading control.

Statistical analysis. Statistical analyses were performed using a one-way analysis of variance or a Kruskal-Wallis non-parametric test, according to the Gaussian or non-Gaussian distribution of the data. $\mathrm{P}<0.05$ was considered to indicate a statistically significant difference in miRNA expression profiling. All data are presented as the mean \pm standard deviation. Statistical analysis was performed using SPSS software 17.00 (SPSS Inc., Chicago, IL, USA). 
$\mathbf{A}$

3'UTR rat smad 7

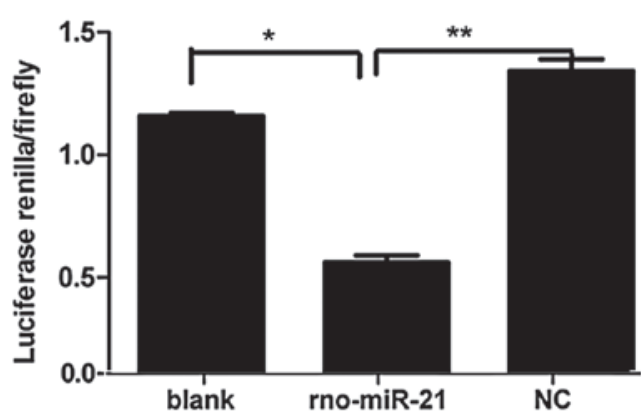

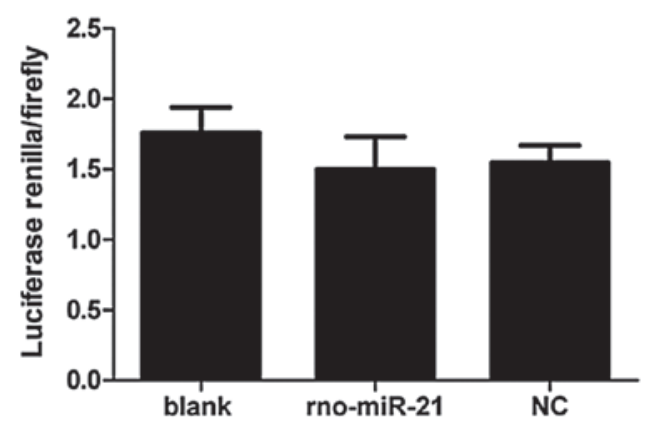

Figure 2. A luciferase assay confirmed that SMAD7 could be regulated by miR-21. (A) The miR-21 transfected group showed a lower luciferase renilla/firefly expression compared with that in the blank and NC groups transfected with 3UTR' rat SMAD7 ("P $<0.05$ and $\left.{ }^{* *} \mathrm{P}<0.01\right)$. (B) There were no significant differences between the the luciferase renilla/firefly expression in the miR-21, blank and NC group in HEK 293T cells cotransfected with the mutant rat SMAD7 vector $(\mathrm{P}>0.05)$.
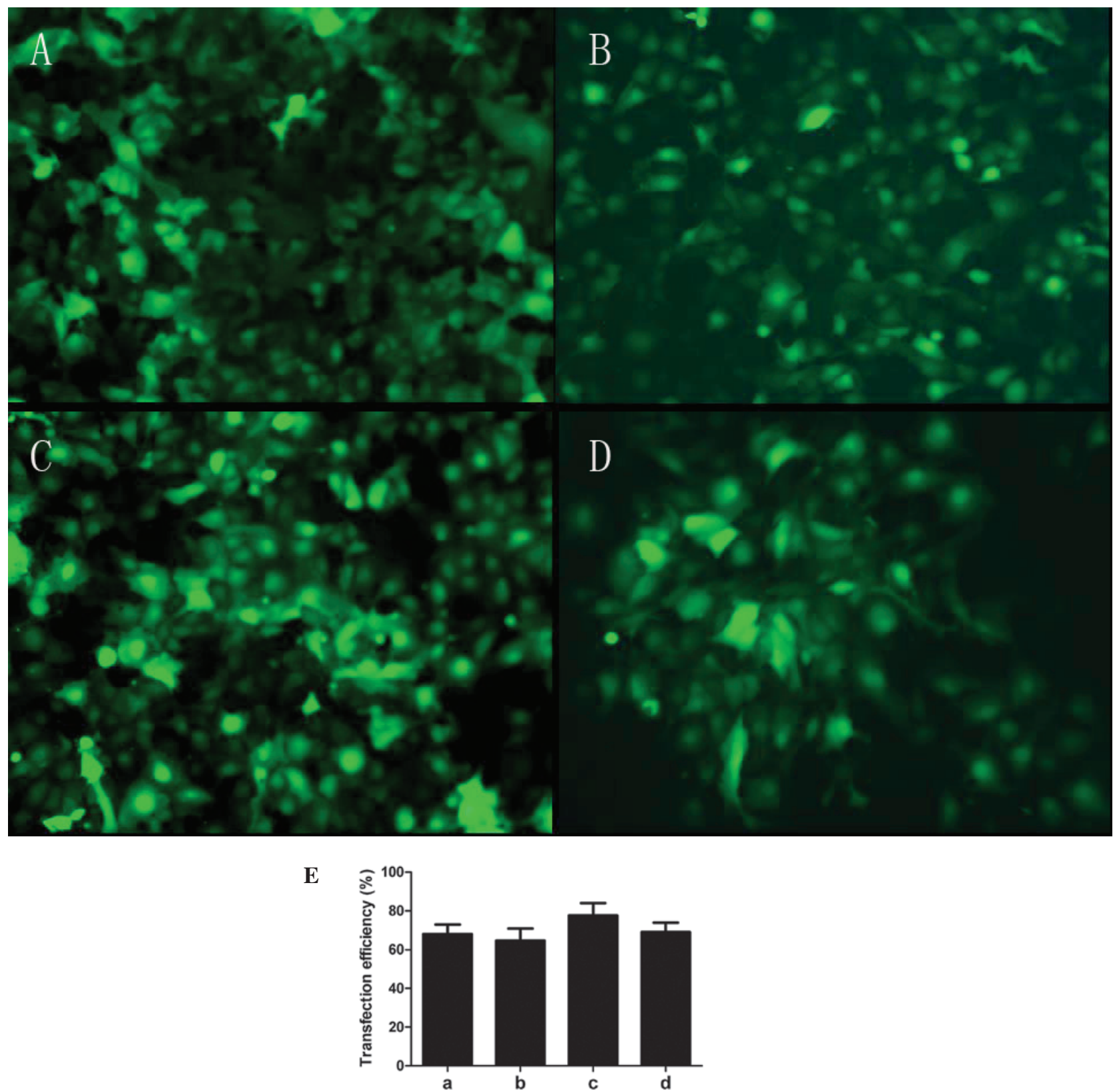

Figure 3. miR-21 lentivirus transfection efficiency following $72 \mathrm{~h}$ incubation. (A) Low glucose empty virus control group. (B) Low glucose group transfected with lentivirus overexpressing miR-21. (C) High glucose empty virus control group. (D) High glucose group transfected with lentivirus overexpressing miR-21. Images were captured at x200 magnification. (E) Transfection efficiency quantification across groups A-D (\%). 


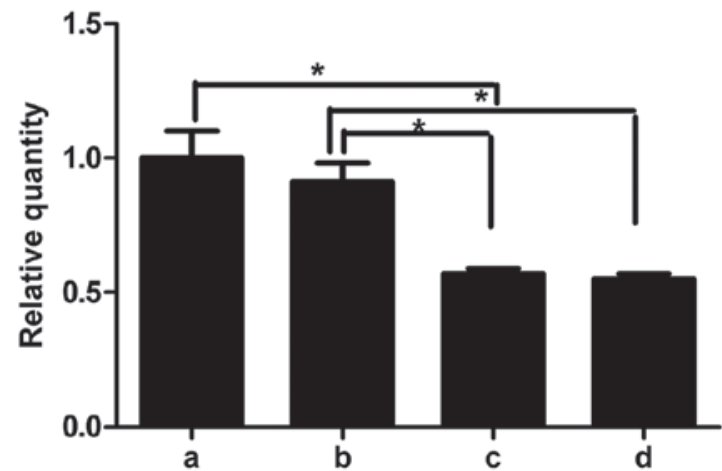

Figure 4. Expression of miR-21 was higher in rat renal tubular epithelial cells with high glucose transfected with lentivirus overexpressing miR-21 as compared with the high glucose empty virus control group $(\mathrm{P}<0.05)$. a, low glucose cell control group without virus transfection; $b$, high glucose group transfected with lentivirus overexpressing miR-21; c, high glucose cell control group without virus transfection; and d, high glucose group with empty virus transfection.

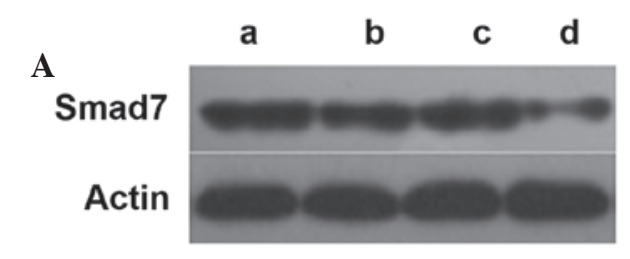

B

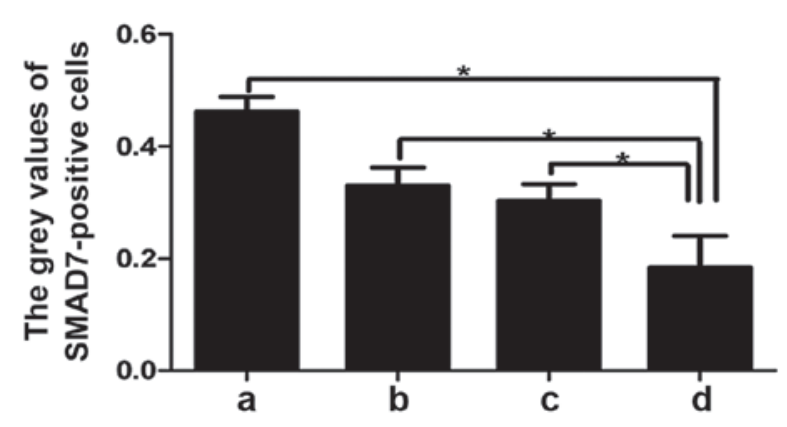

Figure 5. SMAD7 protein expression in rat renal tubular epithelial cells $72 \mathrm{~h}$ after transfection with miR-21 lentivirus. SMAD7 protein expression was lower as compared with the empty lentivirus transfected group, and the untransfected low glucose and high glucose control groups $(\mathrm{P}<0.05)$. a, low glucose cell control group without virus transfection; $b$, high glucose cell control group without virus transfection; c, high glucose control group transfected with empty virus; d, high glucose group transfected with lentivirus overexpressing miR-21.

\section{Results}

$S M A D 7$ is a predicted target of $m i R-21$. In order to determine whether SMAD7 was a predicted target of miR-21, the miRNA GenBank was used. Using the TargetScan software, it was predicted that SMAD7 was a target of miR-21. One binding site for miR-21 was identified in the 3'UTR of the SMAD7 gene, complementary to the 3 ' region of miR-21. The predicted binding site was located at 1192-1198 and contained seven conservative target sites (Fig. 1).

Luciferase assay confirms that SMAD7 is regulated by miR-21. To confirm whether SMAD7 could be regulated by miR-21

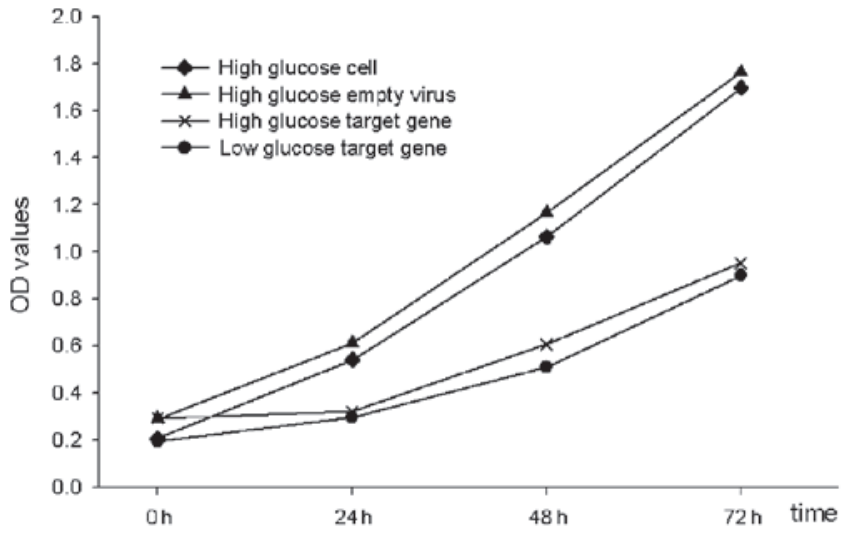

Figure 6. Cellular proliferation in the four groups, a high glucose control group without virus transfection, a high glucose group transfected with empty virus and high and low glucose groups transfected with the lentivirus overexpressing miR-21.

in vitro, wild-type and mutant plasmids expressing the 3 'UTR region predicted to bind miR-21, were constructed. Then miR-21-mimics with a mutated seed region and miR-21-negative control (NC) were synthetized (Shanghai Jima Company, Shanghai, China). Subsequently, the two luciferase reporter vectors with miR-21 response were cotransfected into HEK 293 T cells using a Dual-Luciferase Reporter Assay system. As shown in Fig. 2A, following transfection in the HEK 293T cells, a significant difference was observed in luciferase activity between the miR-21 and blank groups $(\mathrm{P}<0.05)$ and between the miR-21 and $\mathrm{NC}$ groups $(\mathrm{P}<0.01)$. These data showed that miR-21 could interact with the 3'UTR of SMAD7, which was not observed in the $\mathrm{NC}$ and blank groups $(\mathrm{P}>0.05)$. As shown in Fig. 2B, in HEK 293T cells cotransfected with the mutated 3'UTR rat SMAD7 gene, there was no statistical significance among the miR-21, blank and $\mathrm{NC}$ groups $(\mathrm{P}>0.05)$. These data indicate that the SMAD7 3'UTR could not interact with miR-21 when mutated. Therefore, these results confirm that the SMAD7 3'UTR may be regulated by miR-21.

Protein level of SMAD7 inversely correlates with the expression level of miR-21 in rat renal tubular epithelial cells. To further confirm whether miR-21 directly targets the 3'UTR of SMAD7, lentiviral vectors overexpressing miR-21 and empty lentiviral vectors were constructed. The plasmids were transfected into rat renal tubular epithelial cells respectively (Fig. 3) and the transfection efficiency was analyzed using flow cytometry (Fig. 3E). The RNA and protein was extracted from renal tubular epithelial cells, $72 \mathrm{~h}$ post-transfection. The results of RT-qPCR demonstrated that the expression of miR-21 was higher in the lentivirus-transfected rat renal tubular epithelial cells (Fig. 4b) cultured in high glucose compared with untransfected cells cultured in high glucose (Fig.4c). There was no difference between the transfected and untransfected cells in the low glucose groups (data not shown). By western blot analysis, it was shown that the protein expression of SMAD7 was increased in the low glucose groups. In the high glucose groups, the SMAD7 expression was lower compared with that in the low glucose group. When lentivirus expressing miR-21 and empty lentivirus were transfected the protein expression of SMAD7 was lower in the miR-expressing groups in high 
and low glucose conditions (Fig. 5) when compared with the cells transfected with empty lentivirus.

miR-21 inhibits the proliferation of rat renal tubular epithelial cells. To investigate the effects of miR-21 on renal tubular epithelial cells, miR-21 overexpressing- and empty lentiviruses were transfected into the rat renal tubular epithelial cells and were cultured under high and low glucose conditions. Following transfection for 24,48 and $72 \mathrm{~h}$, it was detected that the cellular proliferation in the miR-21-transfected lentivirus group was significantly decreased compared with the empty lentivirus and untransfected groups $(\mathrm{P}<0.01)$. These data indicated that overexpression of miR-21 may inhibit rat renal tubular epithelial cellular proliferation (Fig. 6).

\section{Discussion}

miRNA are a class of non-coding, highly conserved RNA molecules, with numerous biological functions in various processes, including development, differentiation, cell proliferation and apoptosis $(25,26)$.

TGF- $\beta$ is a member of a large family of structurally related cytokines, including activins, Nodal and bone morphogenetic proteins, which transmit cellular signals through the Smad signaling pathway (27). TGF- $\beta 1$ binds to TGF- $\beta$ receptor II (T $\beta$ RII), to initiate intracellular signaling. This results in activation of T $\beta$ RI kinase, which phosphorylates and activates Smad 2 and 3. Activated Smad 2 and 3 form heteromeric complexes, together with Smad4, and translocate to the nucleus to regulate target gene activity. Inhibitory Smads (SMAD6 and SMAD7) provide negative feedback and repress TGF- $\beta$ superfamily signaling through various molecular mechanisms (28).

miRNA-21 is a conserved miRNA that has been associated with various types of tumors. Less research has been performed on the cellular pathways involving miR-21 in renal diseases. Certain studies have shown that miRNAs are associated with TGF- $\beta$ signaling in other diseases $(29,30)$. This study has explored the possible role of miR-21 in TGF- $\beta$ signaling, and confirmed the results of previous studies that showed SMAD7 to be a direct target of miR-21, with predicted target sites in its 3'UTR. To further confirm whether SMAD7 could be regulated by miR-21, a luciferase assay was used to assess the SMAD7 3'UTR reporter activity when co-transfected with miR-21. Mutation in the SMAD7 miR-21 target site reduced the inihibitory effects on SMAD7 expression. When miR-21 lentivirus was transfected into rat renal tubular epithelial cells, the protein level of SMAD7 was lower than that of the empty lentivirus-transfected cells, in untransfected low glucose and high glucose cells conditions.

These data confirmed that SMAD7 is a direct target of miR-21, and that miR-21 can repress the expression of SMAD7 proteins and inhibit the proliferation of rat renal tubular epithelial cells by targeting TGF- $\beta /$ SMAD signaling in vitro. Preliminary data suggested that miR-21 affects proliferation of rat renal tubual epithelial cells and further studies should investigate whether miR-21 is abberantly expressed in renal disease. Further studies using a diabetic nephropathy animal model are required in order to determine the function of miR-21 regulation of SMAD7 in diabetes.

\section{Acknowledgements}

The authors would like to thank the teachers, including Professor Weixue Tang, of the First Affiliated Hospital Chongqing Medical University Experiment Center, for their assistance in the experimental studies.

\section{References}

1. Baek D, Villén J, Shin C, et al: The impact of microRNAs on protein output. Nature 455: 64-71, 2008

2. Ambros V: The functions of animal microRNAs. Nature 431: 350-355, 2004

3. Bartel DP: MicroRNAs: genomics, biogenesis, mechanism, and function. Cell 116: 281-297, 2004.

4. Eulalio A, Huntzinger E and Izaurralde E: Getting to the root of miRNA-mediated gene silencing. Cell 132: 9-14, 2008.

5. Calin GA, Dumitru CD, Shimizu M, Bichi R, et al: Frequent deletions and down-regulation of microRNA genes miR15 and miR16 at 13q14 in chronic lymphocytic leukemia. Proc Natl Acad Sci USA 99: 15524-15529, 2002.

6. Calin GA, Ferracin M, Cimmino A, Di Leva G, et al: A MicroRNA signature associated with prognosis and progression in chronic lymphocytic leukemia. N Engl J Med 353: 1793-1801, 2005.

7. Michael MZ, O'Conner SM, van Holst Pellekaan NG, Young GP and James RJ: Reduced accumulation of specific microRNAs in colorectal neoplasia. Mol Cancer Res 1: 882-891, 2003.

8. Pfeffer S, Zavolan M, Grasser FA, Chien M, et al: Identification of virus-encoded microRNAs. Science 304: 734-736, 2004.

9. Hwang $\mathrm{H}$ and Mendell J: MicroRNAs in cell proliferation, cell death and tumorigenesis. Br J Cancer 94: 776-780, 2006.

10. Chen C, Li L, Lodish H and Bartel D: MicroRNAs modulate hematopoietic lineage differentiation. Science 303: 83-86, 2004.

11. Chan JA, Krichevsky AM and Kosik KS: MicroRNA-21 is an antiapoptotic factor in human glioblastoma cells. Cancer Res 65: 6029-6033, 2005.

12. Yanaihara N, Caplen N, Bowman E, Seike M, et al: Unique microRNA molecular profiles in lung cancer diagnosis and prognosis. Cancer Cell 9: 189-198, 2006.

13. Markou A, Tsaroucha EG, Kaklamanis L, et al: Prognostic value of mature microRNA-21 and microRNA-205 overexpression in non-small cell lung cancer by quantitative real-time RT-PCR. Clin Chem 54: 1696-1704, 2008.

14. Volinia S, Calin GA, Liu CG, Ambs S, et al: A microRNA expression signature of human solid tumors defines cancer gene targets. Proc Natl Acad Sci USA 103: 2257-2261, 2006.

15. Slaby O, Svoboda M, Fabian P, Smerdova T, et al: Altered expression of miR-21, miR-31, miR-143 and miR-145 is related to clinicopathologic features of colorectal cancer. Oncology 72: 397-402, 2007.

16. Schetter AJ, Leung SY, Sohn JJ, Zanetti KA, et al: MicroRNA expression profiles associated with prognosis and therapeutic outcome in colonadenocarcinoma. JAMA 299: 425-436, 2008.

17. Meng F, Henson R, Lang M, Wehbe H, et al: Involvement of human micro-RNA in growth and response to chemotherapy in human cholangiocarcinoma cell lines. Gastroenterology 130: 2113-2129, 2006.

18. Papadopoulos GL, Reczko M, Simossis VA, Sethupathy P and Hatzigeorgiou AG: The database of experimentally supported targets: a functional update of TarBase. Nucleic Acids Res 37: D155-D158, 2009.

19. Selcuklu SD, Donoghue MT, and Spillane C: miR-21 as a key regulator of oncogenic processes. Biochem Soc Trans 37: 918-925, 2009.

20. Ashcroft GS, Yang X, Glick AB, Weinstein M, et al: Mice lacking Smad3 show accelerated wound healing and an impaired local inflammatory response. Nat Cell Biol 1: 260-266, 1999.

21. Attisano L, Wrana JL: Smads as transcriptional co-modulators. Curr Opin Cell Biol 12: 235-243, 2000.

22. Attisano L, Wrana JL: Signal transduction by the TGF-beta superfamily. Science 296: 1646-1647, 2002.

23. Hayashi H, Abdollah S, Qiu Y, et al: The SMAD-related protein SMAD7 associates with the TGF beta receptor and functions as an antagonist of TGF beta signaling. Cell 89: 1165-1173, 1997. 
24. Kavsak P, Rasmussen RK, Causing CG, et al: SMAD7 binds to Smurf 2 to form an E3 ubiquitin ligase that targets the TGF beta receptor for degradation. Mol Cell 6: 1365-1375, 2000.

25. Karp X, Ambros V: Encountering microRNAs in cell fate signaling. Science 310: 1288-1289, 2005.

26. Chen CZ, Li L, Lodish HF and Bartel DP: MicroRNAs modulate hematopoietic lineage differentiation. Science 303: 83-86, 2004.

27. Heldin $\mathrm{CH}$, Miyazono K and ten Dijke P: TGF-beta signaling from cell membrane to nucleus through SMAD proteins. Nature 390: 465-471, 1997.
28. Massague $\mathrm{J}$ and Wotton D: Transcriptional control by the TGF-beta/Smad signaling system. EMBO J 19: 1745-1754, 2000. 29. Zhu H, Li Y, Qu S, et al: MicroRNA expression abnormalities in limited cutaneous scleroderma and diffuse cutaneous scleroderma. J Clin Immunol 32: 514-522, 2012.

30. Wei J, Feng L, Li Z, Xu G and Fan X: MicroRNA-21 activates hepatic stellate cells via PTEN/Akt signaling. Biomed Pharmacother 67: 387-392, 2013. 